\title{
Observers Design for Singular Fractional-Order Systems
}

\author{
Ibrahima N'Doye, Mohamed Darouach, Michel Zasadzinski and Nour-Eddine Radhy
}

\begin{abstract}
This paper considers the observers design for continuous-time singular fractional-order systems. The approach is based on the generalized Sylvester equations solutions. The conditions for the existence of these observers are given and sufficient conditions for their stability are derived in terms of linear matrix inequalities formulation.

Index Terms - Singular fractional-order systems, generalized Sylvester equations, linear matrix inequality (LMI), reduced order observer, minimal order observer, full order observer, stability.
\end{abstract}

\section{INTRODUCTION}

State estimation or observer design have been widely used in control and signal processing in the last few decades. They are of theoretical interest and also have some applications particularly in the failure detection and fault diagnosis problems, and chaotic synchronization and secure communications [1], [2], [3], [4], [5]. The problem of observer design for linear and singular systems has been greatly treated for the standard and singular systems with or without unknown inputs [6], [7], [2].

Fractional-order systems (i.e. systems containing fractional derivatives and/or integrals) have been studied by many authors in engineering sciences from an application point of view (see [8], [9] and references therein). Many systems can be described with the help of fractional derivatives. These systems are known to display fractional-order dynamics : electromagnetic systems [10], [11], dielectric polarization [12], viscoelastic systems [13], [14] chaotic synchronization and secure communications.

Singular systems (known as generalized, descriptor of differential algebraic systems) describe a large class of systems, which are not only theoretical interest but also have a great importance in practice [3]. They are encountered in chemical and mineral industries, for example the dynamic balances of mass and energy are described by differential equations while thermodynamic equilibrium relations constitute additional algebraic constraints. The problem of the state estimation for these practical applications arises in data

Ibrahima N'Doye is with Nancy Université, Centre de Recherche en Automatique de Nancy (CRAN UMR-7039, CNRS), IUT de Longwy, 186 rue de Lorraine 54400 Cosnes et Romain, France and with Université Hassan II, Faculté des Sciences Ain-Chock, Laboratoire Physique et Matériaux Microélectronique Automatique et Thermique BP: 5366 Maarif, Casablanca 20100, Morocco

Mohamed Darouach and Michel Zasadzinski are with Nancy Université, Centre de Recherche en Automatique de Nancy (CRAN UMR-7039, CNRS), IUT de Longwy, 186 rue de Lorraine 54400 Cosnes et Romain, France

Nour-Eddine Radhy is with Université Hassan II, Faculté des Sciences Ain-Chock, Laboratoire Physique et Matériaux Microélectronique Automatique et Thermique BP: 5366 Maarif, Casablanca 20100, Morocco reconciliation for example [4]. Singular systems are also frequently encountered in electronic and economics [15].

The question of stability is crucial in control theory. In the field of fractional-order control systems, there are many challenging and unsolved problems related to stability theory such as robust stability, bounded input-bounded output stability, internal stability, etc. Stability results on fractionalorder control systems have been presented in [16], [17], [18].

In our knowledge, the present work is the first one presenting in an unified framework the observers design for singular fractional-order systems. The approach is based on the parameterization of the generalized Sylvester equations solutions and the design of reduced order, minimal order and full order observers for singular fractional-order systems are presented in a compact formulation. The conditions for the existence of these observers are given, necessary and sufficient conditions for their stability are derived in terms of linear matrix inequalities formulation with fractional-order $\alpha$ belonging to $0<\alpha<2$.

This paper is organized as follows.

In section II, we provide some background on the fractional derivative, the observability and the stability of fractional-order systems with the fractional-order $\alpha$ belonging to $0<\alpha<2$.

In section III we present the system description and formulate the observers design problem.

Section IV presents the main results of the paper. Necessary and sufficient conditions for the asymptotical stability of observers with fractional-order $\alpha$ belonging to $0<\alpha \leqslant 1$ and $1 \leqslant \alpha<2$ are presented.

Notations. $M^{T}$ is the transpose of $M, \operatorname{Sym}\{X\}$ is used to denote $X^{T}+X, \Omega^{+}$is any generalized inverse of $\Omega$ satisfying $\Omega \Omega^{+} \Omega=\Omega$ and $D^{\alpha}$ represents initialized $\alpha^{\text {th }}$ order differintegration.

\section{Preliminaries Definitions}

\section{A. The fractional derivative}

Formulations of noninteger-order derivatives fall into two main classes: the Riemann-Liouville derivative and the Grûnward-Letnikov derivative, on one hand, defined as [8], [19]

$D^{\alpha} f(t)=\frac{1}{\Gamma(n-\alpha)} \frac{\mathrm{d}^{n}}{\mathrm{~d} t^{n}} \int_{a}^{t} \frac{f(\tau)}{(t-\tau)^{\alpha-n+1}} \mathrm{~d} \tau, \quad n-1 \leqslant \alpha<n$

or the Caputo derivative on the other, defined as [19], [20]

$$
D^{\alpha} f(t)=\frac{1}{\Gamma(n-\alpha)} \int_{a}^{t} \frac{\frac{\mathrm{d}^{n} f(\tau)}{\mathrm{d} t^{n}}}{(t-\tau)^{\alpha-n+1}} \mathrm{~d} \tau, \quad n-1 \leqslant \alpha<n
$$


with $n \in \mathbb{N}$ and $\alpha \in \mathbb{R}^{+}$, where $\Gamma($.$) is the Gamma$ function. The physical interpretation of the fractional derivatives and the solution of fractional differential equations are given in [19], [20]. Here and throughout the paper, only the Caputo definition is used since this Laplace transform allows utilization of initial values of classical integer-order derivatives with clear physical interpretations. In the rest of the paper, $D^{\alpha}$ is used to denote the Caputo fractional derivative of order $\alpha$.

Consider the following linear fractional-order systems

$$
\begin{cases}D^{\alpha} x(t)=A x(t)+B u(t) & \\ y(t)=C x(t) & 0<\alpha<2 \\ x(0)=x_{0} & \end{cases}
$$

where $x(t) \in \mathbb{R}^{n}$ is the state vector, $u(t) \in \mathbb{R}^{m}$ is the control input vector and $y(t) \in \mathbb{R}^{p}$ is the measured output. $A, B$ and $C$ are known constant matrices.

\section{B. Stability of fractional-order systems}

The stability domain of fractional-order linear system with order $1 \leqslant \alpha<2$ is a convex set that can be described by LMI methods, and the stability domain for the case $0<\alpha \leqslant 1$ is a nonconvex set that can not described by LMI methods. However, in the case where $0<\alpha \leqslant 1$, the formulation introduced in [21] and developed in [22] permits to treat this particular case using the concept of Generalized LMI $(\mathcal{G L M I})$ regions. This concept is based on the fact that the stability domain can be viewed as the union of two half planes and is initially extended in the case of fractional-order systems in [23].

Definition 1: It has been shown that system (3) is asymptotically stable if the following condition is satisfied [16], [24] for $0<\alpha \leqslant 1$, [25] for $1 \leqslant \alpha<2$

$$
|\arg (\operatorname{spec}(A))|>\alpha \frac{\pi}{2}
$$

where $\operatorname{spec}(A)$ represents the eigenvalues of matrix $A$.

The stability of matrix $A$ is equivalent to the stability of matrix $A^{T}$, then the necessary and sufficient LMIs conditions to satisfy condition (4) when the fractional-order $\alpha$ belonging to $1 \leqslant \alpha<2$ and $0<\alpha \leqslant 1$ are given in the following lemmas.

Lemma 1: [25], [23], [26] Let $A^{T} \in \mathbb{R}^{n \times n}$ the transpose of matrix $A$. Then, the fractional-order system $D^{\alpha} x(t)=$ $A x(t)$ is asymptotically stable (i.e. $|\arg (\operatorname{spec}(A))|>\alpha \frac{\pi}{2}$ where $1 \leqslant \alpha<2$, if and only if there exits $P_{0}=P_{0}^{T}>0$ such that

$$
\left[\begin{array}{cc}
\left(P_{0} A+A^{T} P_{0}\right) \sin \theta & -\left(P_{0} A-A^{T} P_{0}\right) \cos \theta \\
\left(P_{0} A-A^{T} P_{0}\right) \cos \theta & \left(P_{0} A+A^{T} P_{0}\right) \sin \theta
\end{array}\right]<0
$$

where $\theta=\pi-\alpha \frac{\pi}{2}$.

Lemma 2: [27], [28] Let $A^{T} \in \mathbb{R}^{n \times n}$ the transpose of matrix $A$. The fractional-order system $D^{\alpha} x(t)=A x(t)$ is asymptotically stable $\left(\right.$ i.e. $\left.|\arg (\operatorname{spec}(A))|>\alpha \frac{\pi}{2}\right)$ where $0<$ $\alpha \leqslant 1$, if and only if there exist two real symmetric matrices $P_{k 1} \in \mathbb{R}^{n \times n}, k=1,2$, and two skew-symmetric matrices $P_{k 2} \in \mathbb{R}^{n \times n}, k=1,2$, such that

$$
\sum_{i=1}^{2} \sum_{j=1}^{2} \operatorname{Sym}\left\{\Gamma_{i j} \otimes\left(A^{T} P_{i j}\right)\right\}<0
$$

$$
\left[\begin{array}{cc}
P_{11} & P_{12} \\
-P_{12} & P_{11}
\end{array}\right]>0, \quad\left[\begin{array}{cc}
P_{21} & P_{22} \\
-P_{22} & P_{21}
\end{array}\right]>0,
$$

where

$$
\begin{aligned}
& \Gamma_{11}=\left[\begin{array}{cc}
\sin \left(\alpha \frac{\pi}{2}\right) & -\cos \left(\alpha \frac{\pi}{2}\right) \\
\cos \left(\alpha \frac{\pi}{2}\right) & \sin \left(\alpha \frac{\pi}{2}\right)
\end{array}\right], \Gamma_{12}=\left[\begin{array}{cc}
\cos \left(\alpha \frac{\pi}{2}\right) & \sin \left(\alpha \frac{\pi}{2}\right) \\
-\sin \left(\alpha \frac{\pi}{2}\right) & \cos \left(\alpha \frac{\pi}{2}\right)
\end{array}\right], \\
& \Gamma_{21}=\left[\begin{array}{cc}
\sin \left(\alpha \frac{\pi}{2}\right) & \cos \left(\alpha \frac{\pi}{2}\right) \\
-\cos \left(\alpha \frac{\pi}{2}\right) & \sin \left(\alpha \frac{\pi}{2}\right)
\end{array}\right], \Gamma_{22}=\left[\begin{array}{cc}
-\cos \left(\alpha \frac{\pi}{2}\right) & \sin \left(\alpha \frac{\pi}{2}\right) \\
-\sin \left(\alpha \frac{\pi}{2}\right) & -\cos \left(\alpha \frac{\pi}{2}\right)
\end{array}\right] .
\end{aligned}
$$

The two drawings of the figures below illustrate the stability region of linear fractional-order systems with fractional order belonging to $0<\alpha \leqslant 1$ and $1 \leqslant \alpha<2$.

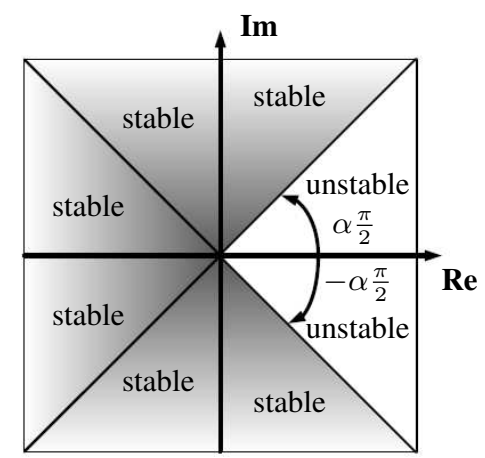

Fig. 1. Stability region of linear fractional-order systems with order $0<$ $\alpha \leqslant 1$.

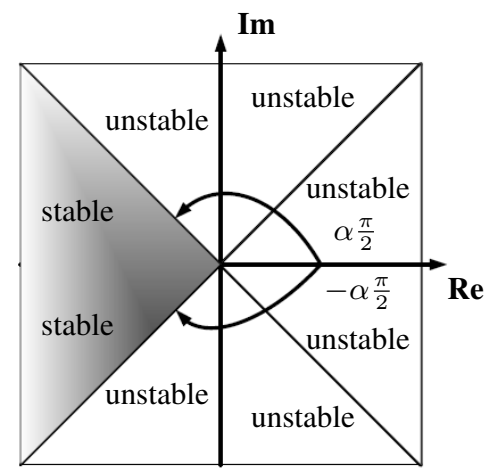

Fig. 2. Stability region of linear fractional-order systems with order $1 \leqslant$ $\alpha<2$.

\section{Detectability of fractional-order systems}

Lemma 3: [29], [30] System (3) is detectable by an asymptotic observer if the unobservable modes of matrix $A$ are asymptotically stable.

Proof: The proof of lemma 3 can be established as in the usual integer order case, since it involves algebraic properties of the pair $(C, A)$ only.

Remark 1: [29], [30] In particular if $(C, A)$ is observable, the spectrum of $\mathbb{L}=A-L C$ can be assigned anywhere in the complex region of asymptotic stability (i.e. $\left.|\arg (\operatorname{spec}(\mathbb{L}))|>\alpha \frac{\pi}{2}\right)$. 


\section{SYSTEM DESCRIPTION AND OBSERVER FORM}

Consider the following linear descriptor fractional-order systems

$$
\begin{cases}E D^{\alpha} x(t)=A x(t)+B u(t) & \\ y(t)=C x(t) & 0<\alpha<2 \\ x(0)=x_{0} & \end{cases}
$$

where $x(t) \in \mathbb{R}^{n}$ is the descriptor vector, $u(t) \in \mathbb{R}^{m}$ is the control input vector and $y(t) \in \mathbb{R}^{p}$ is the measured output. Matrix $E \in \mathbb{R}^{n_{E} \times n}$ and when $n_{E}=n$ matrix $E$ is singular, $A \in \mathbb{R}^{n_{E} \times n}, B \in \mathbb{R}^{n_{E} \times m}$ and $C \in \mathbb{R}^{p \times n}$. Let $\Phi \in \mathbb{R}^{r_{1} \times n_{E}}$ be a full row rank matrix such that $\Phi E=0$, then, from (8) we have

$$
\Phi B u(t)=-\Phi A x(t)
$$

Assumption 1: Assume that

$$
\operatorname{rank}\left[\begin{array}{c}
E \\
\Phi A \\
C
\end{array}\right]=n
$$

As in [2], [3], it is easy to see that assumption 1 is equivalent to the impulse observability, i.e.

$\operatorname{rank}\left[\begin{array}{cc}E & A \\ 0 & E \\ 0 & C\end{array}\right]=\operatorname{rank}\left[\begin{array}{c}E \\ \Phi A \\ C\end{array}\right]+\operatorname{rank} E=n+\operatorname{rank} E$

Now, consider the following reduced-order observer for system (8)

$$
\left\{\begin{array}{l}
D^{\alpha} \eta(t)=N \eta(t)+J y(t)+H u(t) \\
\widehat{x}(t)=P \widehat{x}(t)-Q \Phi B u(t)+F y(t) \quad 0<\alpha<2 \\
\eta(0)=\eta_{0}
\end{array}\right.
$$

where $\eta(t) \in \mathbb{R}^{q}$ is the state vector of observer and $\widehat{x}(t) \in$ $\mathbb{R}^{n}$ is the estimate of $x(t)$. Matrices $N, J, H, P, Q$, and $F$ are unknown matrices of appropriate dimensions, which must be determined such that $\widehat{x}(t)$ asymptotically converges to $x(t)$.

The following proposition gives the conditions for the existence and stability of observer (9).

Proposition 1: Under assumption 1, system (9) is an asymptotic observer, i.e. $\lim _{t \rightarrow \infty} \widehat{x}(t)-x(t)=0$ where $0<$ $\alpha<2$, if there exits a matrix $T$ such that

i) $D^{\alpha} \varepsilon(t)=N \varepsilon(t)$ is asymptotically stable,

ii) $N T E-T A+J C=0$,

iii) $H=T B$,

iv) $\left[\begin{array}{lll}P & Q & F\end{array}\right]\left[\begin{array}{c}T E \\ \Phi A \\ C\end{array}\right]=I_{n}$.

Proof: Define $\varepsilon(t)=\eta-T E x(t)$, the error between $\eta$ and $\operatorname{TEx}(t)$, then its fractional-order dynamic is given by

$$
D^{\alpha} \varepsilon(t)=D^{\alpha} \eta(t)-T E D^{\alpha} x(t)
$$

or equivalently

$D^{\alpha} \varepsilon(t)=N \varepsilon(t)+(N T E+J C-T A) x(t)+(H-T B) u(t)$.

On the other hand from the definition of $\varepsilon(t)$, we have

$$
\widehat{x}(t)=P \varepsilon(t)+\left[\begin{array}{lll}
P & Q & F
\end{array}\right]\left[\begin{array}{c}
T E \\
\Phi A \\
C
\end{array}\right] x(t) .
$$

If items i) - iii) of the proposition are satisfied then $\lim _{t \rightarrow \infty} \eta-T E x(t)=0$.

In addition if item iv) is satisfied, then $\lim _{t \rightarrow \infty} \widehat{x}(t)-x(t)=$ $\lim _{t \rightarrow \infty} e(t)=\lim _{t \rightarrow \infty} P \varepsilon(t)=0$.

Then, the estimation error is independent of $u(t)$ and $x(t)$, and we obtain the following observer error system

$$
\left\{\begin{array}{l}
D^{\alpha} \varepsilon(t)=N \varepsilon(t) \\
e(t)=P \varepsilon(t)
\end{array} \quad 0<\alpha<2\right.
$$

Items ii) - iv) correspond to a constrained generalized Sylvester equation, of proposition 1 of assumption 1, let $\Psi$ be an arbitrary matrix and define the following matrix $T^{\prime}=T-\Psi \Phi$, then this constrained generalized Sylvester equation becomes [3]

$$
\left[\begin{array}{ccc}
N & -\Psi & J \\
P & Q & F
\end{array}\right]\left[\begin{array}{c}
T^{\prime} E \\
\Phi A \\
C
\end{array}\right]=\left[\begin{array}{c}
T^{\prime} A \\
I_{n}
\end{array}\right]
$$

where we have used the fact that $\Phi E=0$.

Equation (14) have a solution if only if

$$
\operatorname{rank}\left[\begin{array}{c}
T^{\prime} E \\
\Phi A \\
C \\
T^{\prime} A \\
I_{n}
\end{array}\right]=\operatorname{rank}\left[\begin{array}{c}
T^{\prime} E \\
\Phi A \\
C
\end{array}\right]=n=\operatorname{rank}\left[\begin{array}{c}
E \\
\Phi A \\
C
\end{array}\right] .
$$

The design of observer of dimension $q$ is reduced to finding the matrices $T^{\prime}, N, \Psi, J, H, P, Q$, and $F$ such that equation (14) and items i) - iii) of proposition 1 are satisfied.

Remark 2: One can see that the dimension of observer (9) is of dimension $q \leqslant n$. Then, the presented approach unifies the observers design for the full order $q=n$, the reduced order $q=n-p$ and the minimal order observers

\section{MAin RESUlts}

In this section, we give sufficient conditions for the existence and stability of observer (9).

The method to design observer (9) for system and its existence and stability conditions are given in [3], [31]. From proposition 1, the design of observer (9) of dimension $q$ is reduced to find the matrices $T, N, J, H, P$, and $Q$ such that items i) - iv) of proposition 1 are satisfied.

The following lemma shows how we can solve, under assumption 1, the constrained Sylvester equations (14) and 
obtain the expressions of matrices $T^{\prime}, N, \Psi, J, H, P, Q$, and $F$ solution to (14).

Lemma 4: [3], [31] Let $R \in \mathbb{R}^{q \times n}$ be any full row rank matrix and define the matrix $\sum=\left[\begin{array}{c}R \\ \Phi A \\ C\end{array}\right]$ such that $\operatorname{rank} \sum=n$, then under assumption 1, the general solution to (14) is given by

$$
\begin{gathered}
N=\mathbb{A}-Z_{1} \mathbb{B}-Y_{1} \mathbb{C} \\
T=T^{\prime}+\Psi \Phi \\
P=\left[\begin{array}{c}
R \\
\Phi A \\
C
\end{array}\right]^{+}\left[\begin{array}{c}
I_{q} \\
0
\end{array}\right]
\end{gathered}
$$

and

$\left[\begin{array}{cc}-\Psi & J \\ Q & F\end{array}\right]=\left[\begin{array}{c}T^{\prime} A \\ I_{n}\end{array}\right] \Omega^{+}-\left[\begin{array}{c}Y_{1} \\ Y_{2}\end{array}\right]\left(I_{q+r_{1}+p}-\Omega \Omega^{+}\right)\left[\begin{array}{c}K \\ I_{r_{1}+p}\end{array}\right]$

where $T^{\prime}=\Lambda_{1}-Z_{1} \Delta_{1}, K=\Lambda_{2}-Z_{1} \Delta_{2}, \Omega=\left[\begin{array}{c}E \\ \Phi A \\ C\end{array}\right]$,

$\Lambda_{1}=R \Omega^{+}\left[\begin{array}{c}I_{n} \\ 0\end{array}\right], \Lambda_{2}=R \Omega^{+}\left[\begin{array}{c}0 \\ I_{n+p}\end{array}\right]$,

$\Delta_{1}=-\left(I_{n+r_{1}+p}-\Omega \Omega^{+}\right)\left[\begin{array}{c}I_{n} \\ 0\end{array}\right]$,

$\Delta_{2}=-\left(I_{n+r_{1}+p}-\Omega \Omega^{+}\right)\left[\begin{array}{c}0 \\ I_{n+p}\end{array}\right], \mathbb{A}=\Lambda_{1} A \sum^{+}\left[\begin{array}{c}I_{q} \\ 0\end{array}\right]$,

$\mathbb{B}=\Delta_{1} A \sum^{+}\left[\begin{array}{c}I_{q} \\ 0\end{array}\right], \mathbb{C}=\left(I_{q+p+r_{1}}-\sum \sum^{+}\right)\left[\begin{array}{c}I_{q} \\ 0\end{array}\right]$,

and with $Y_{1}$ and $Z_{1}$ are arbitrary matrices of appropriate dimensions.

In these following sections IV-A and IV-B, the conditions for the existence of the parameter matrices $Y_{1}$ and $Z_{1}$ such that the observer (9) is asymptotically stable is giving i.e. condition i) of proposition 1 is realized where $1 \leqslant \alpha<2$ and $0<\alpha \leqslant 1$ respectively.

The problem amounts to study the asymptotical stability for the fractional-order system $D^{\alpha} \varepsilon(t)=N \varepsilon(t)$ because when $\varepsilon(t) \rightarrow 0$, then the estimation error $e(t) \rightarrow 0$ and it's guaranteed the stability of observer (9).

A. Stability of fractional-order error system : The $1 \leqslant \alpha<2$ case

Under assumption 1, the asymptotical stability for the fractional-order observer error system $D^{\alpha} \varepsilon(t)=N \varepsilon(t)$ where $1 \leqslant \alpha<2$ is given in the following theorem.

Theorem 1: Under assumption 1, there exists an asymptotically stable observer of the form (9) where $1 \leqslant \alpha<2$, i.e. condition i) of proposition 1 is satisfied, if and only if there are matrices $W \in \mathbb{R}^{n \times n}$ and $P_{0}=P_{0}^{T}>0 \in \mathbb{R}^{n \times n}$ such that

where

$$
\left[\begin{array}{ll}
\Pi_{11} & \Pi_{12} \\
\Pi_{12}^{T} & \Pi_{22}
\end{array}\right]<0
$$

$\Pi_{11}=\Gamma_{22}=\left(P_{0} \mathbb{A}+\mathbb{A}^{T} P_{0}-W \mathbb{X}-\mathbb{X}^{T} W^{T}\right) \sin \theta$

$\Pi_{12}=\left(\mathbb{A}^{T} P_{0}-P_{0} \mathbb{A}+W \mathbb{X}-\mathbb{X}^{T} W^{T}\right) \cos \theta$

and $W_{1}=W P_{0}^{-1}=\left[\begin{array}{ll}Z_{1} & Y_{1}\end{array}\right], \mathbb{X}=\left[\begin{array}{l}\mathbb{B} \\ \mathbb{C}\end{array}\right]$ with $\theta=$ $\pi-\alpha \frac{\pi}{2}$.

Proof: From lemma 3 and remark 1, one can see that the necessary condition that condition i) of proposition 1 is satisfied, by using (16), that the pair $\left(\left[\begin{array}{l}\mathbb{B} \\ \mathbb{C}\end{array}\right], \mathbb{A}\right)$ is detectable.

Now, suppose that there exist matrices $W \in \mathbb{R}^{n \times n}$ and $P_{0}=P_{0}^{T}>0 \in \mathbb{R}^{n \times n}$ such that (20) holds. It follows from lemma 1 that $|\arg (\operatorname{spec}(N))|>\alpha \frac{\pi}{2}$ is equivalent to

$$
\begin{aligned}
& {\left[\begin{array}{cc}
\left(P_{0} N+N^{T} P_{0}\right) \sin \theta & -\left(P_{0} N-N^{T} P_{0}\right) \cos \theta \\
\left(P_{0} N-N^{T} P_{0}\right) \cos \theta & \left(P_{0} N+N^{T} P_{0}\right) \sin \theta
\end{array}\right]=} \\
& \operatorname{Sym}\left\{\left[\begin{array}{cc}
P_{0} \mathbb{A} \sin \theta & -P_{0} \mathbb{A} \cos \theta \\
P_{0} \mathbb{A} \cos \theta & P_{0} \mathbb{A} \sin \theta
\end{array}\right]\right\} \\
& \quad+\operatorname{Sym}\left\{\left[\begin{array}{cc}
-W \mathbb{X} \sin \theta & W \mathbb{X} \cos \theta \\
-W \mathbb{X} \cos \theta & -W \mathbb{X} \sin \theta
\end{array}\right]\right\}<0
\end{aligned}
$$

where $W_{1}=W P_{0}^{-1}=\left[\begin{array}{ll}Z_{1} & Y_{1}\end{array}\right], \mathbb{X}=\left[\begin{array}{l}\mathbb{B} \\ \mathbb{C}\end{array}\right]$ and $\theta=$ $\pi-\alpha \frac{\pi}{2}$.

Inequality (21) is equivalent to (20). This ends the proof of theorem 1 .

B. Stability of fractional-order error system : The $0<\alpha \leqslant 1$ case

Under assumption 1, the asymptotical stability for the fractional-order observer error system $D^{\alpha} \varepsilon(t)=N \varepsilon(t)$ where $0<\alpha \leqslant 1$ is given in the following theorem.

Theorem 2: Under assumption 1, there exists an asymptotically stable observer of the form (9) where $0<\alpha \leqslant 1$, i.e. condition i) of proposition 1 is satisfied, if and only if there are matrices $L \in \mathbb{R}^{n \times n}$ and $P_{0}=P_{0}^{T}>0 \in \mathbb{R}^{n \times n}$ such that

$$
\sum_{i=1}^{2}\left(\operatorname{Sym}\left\{\Gamma_{i 1} \otimes\left(\mathbb{A}^{T} P_{0}\right)\right\}-\operatorname{Sym}\left\{\Gamma_{i 1} \otimes\left(\mathbb{X}^{T} L\right)\right\}\right)<0
$$

where $\Gamma_{i 1}(i=1,2)$ satisfy $(7), W_{1}^{T}=L P_{0}^{-1}=$ $\left[\begin{array}{ll}Z_{1} & Y_{1}\end{array}\right]^{T}$ and $\mathbb{X}=\left[\begin{array}{l}\mathbb{B} \\ \mathbb{C}\end{array}\right]$.

Proof: From lemma 3 and remark 1, it is easy to see that the necessary condition that condition i) of proposition 1 is satisfied, by using (16), that the pair $\left(\left[\begin{array}{l}\mathbb{B} \\ \mathbb{C}\end{array}\right], \mathbb{A}\right)$ is detectable. 
Now, suppose that there exist matrices $L \in \mathbb{R}^{n \times n}$ and $P_{0}=P_{0}^{T}>0 \in \mathbb{R}^{n \times n}$ such that (22) holds. It follows from lemma 2 that $|\arg (\operatorname{spec}(N))|>\alpha \frac{\pi}{2}$ is equivalent to

$$
\sum_{i=1}^{2} \sum_{j=1}^{2} \operatorname{Sym}\left\{\Gamma_{i j} \otimes\left(N^{T} P_{i j}\right)\right\}<0
$$

where $N=\mathbb{A}-W_{1} \mathbb{X}, W_{1}=\left[\begin{array}{ll}Z_{1} & Y_{1}\end{array}\right], \mathbb{X}=\left[\begin{array}{l}\mathbb{B} \\ \mathbb{C}\end{array}\right]$ and $\Gamma_{i j}(i, j=1,2)$ satisfy (7). By setting $P_{11}=P_{21}=P_{0}$, $P_{12}=P_{22}=0$ in (23), one can conclude that if

$$
\operatorname{Sym}\left\{\Gamma_{11} \otimes\left(N^{T} P_{0}\right)\right\}+\operatorname{Sym}\left\{\Gamma_{21} \otimes\left(N^{T} P_{0}\right)\right\}<0
$$

the fractional-order system $D^{\alpha} \varepsilon(t)=N \varepsilon(t)$ where $0<\alpha \leqslant 1$ is asymptotically stabilizable.

Substitute $N=\mathbb{A}-W_{1} \mathbb{X}$ into (24) and set $L=W_{1}^{T} P_{0}$, we obtain

$$
\sum_{i=1}^{2}\left(\operatorname{Sym}\left\{\Gamma_{i 1} \otimes\left(\mathbb{A}^{T} P_{0}\right)\right\}-\operatorname{Sym}\left\{\Gamma_{i 1} \otimes\left(\mathbb{X}^{T} L\right)\right\}\right)<0
$$

Inequality (25) is equivalent to (22). This completes the proof of theorem 2 .

The algorithm for the observer design is given as follows [3], [31]

\section{Algorithm}

Under assumption 1 the design of the observer (9) can be obtained as follows

- Step 1 : Find a matrix $R$ such that $\sum=\left[\begin{array}{c}R \\ \Phi A \\ C\end{array}\right]$ is a full-column rank.

- Step 2 : Compute $\Lambda_{1}, \Delta_{1}, \Lambda_{2}, \Delta_{2}, \mathbb{A}, \mathbb{B}, P$ and $\mathbb{C}$.

- Step 3 : Solve the LMIs (20) or (22), to deduce the parameter matrices $Z_{1}$ and $Y_{1}$.

- Step 4 : Compute $T^{\prime}=\Lambda_{1}-Z_{1} \Delta_{1}, K=\Lambda_{2}-Z_{1} \Delta_{2}$, $N$ and then deduce $\left[\begin{array}{ll}-\Phi & J\end{array}\right]$ and $\left[\begin{array}{ll}Q & F\end{array}\right]$.

- Step 5 : Compute $T=T^{\prime}+\Psi \Phi$, then deduce $H=T B$.

D. Minimal order, reduced order and full order observers design

In this section, the minimal order, the reduced order and the full order observers are derived with the above results.

1) Minimal order observers

Let $\operatorname{rank} E=r$ and $\Phi \in \mathbb{R}^{r_{1} \times n}$ be a full row rank matrix such that $\Phi E=0$, with $\operatorname{rank} \Phi=r_{1}=n-r=$ $\operatorname{rank} \Phi A$. Then the dimension of the minimal order observer is $q=n-r_{1}-p$, and we have

$$
\sum^{+}=\sum^{-1}=\left[\begin{array}{c}
R \\
\Psi A \\
C
\end{array}\right]^{-1}
$$

From the above results we obtain $\mathbb{C}=0$ and matrix $N$ becomes

$$
N=\mathbb{A}-Z_{1} \mathbb{B}
$$

where $\mathbb{A}=\Lambda_{1} A \sum^{+}\left[\begin{array}{c}I_{q} \\ 0\end{array}\right], \mathbb{B}=\Delta_{1} A \sum^{+}\left[\begin{array}{c}I_{q} \\ 0\end{array}\right]$.

The design of the observer is reduced to the determination of the parameter matrix $Z_{1}$ such that condition i) of proposition 1 is satisfied.

This matrix can be determined by solving the LMIs (20) or (22) with $\mathbb{C}=0$, the rest of the design can be obtained from Algorithm IV-C.

2) Reduced order observers

This case corresponds to the full state estimation using a filter of order $n-p$. It can be obtained when $\Phi=$ 0 , in this case assumption becomes $\operatorname{rank}\left[\begin{array}{c}E \\ C\end{array}\right]=$ $n$. It corresponds to $\Omega=\left[\begin{array}{l}E \\ C\end{array}\right]$ and $\sum=\left[\begin{array}{l}R \\ C\end{array}\right]$ nonsingular then $\Omega^{+}=\Omega^{-1}$.

The reduced order observer design can be obtained as in the case of minimal order presented above.

3) Full order observers

This case corresponds to the state estimation of the full state by using the full order observer. It corresponds to $q=n$ and matrix $R=I_{n}$ then we have, under assumption $1, \operatorname{rank} \Omega=n$ with $\Omega=\left[\begin{array}{c}E \\ \Phi A \\ C\end{array}\right]$ and the following results $\sum^{+}=\left[\begin{array}{c}R \\ \Phi A \\ C\end{array}\right]^{+}=\left[\begin{array}{ll}I_{n} & 0\end{array}\right]$,
$\mathbb{A}=\Lambda_{1} A, \mathbb{B}=\Delta_{1} A, \mathbb{C}=\left[\begin{array}{c}0 \\ \Phi A \\ C\end{array}\right]$ and $P=I_{n}$. Where

$$
\begin{aligned}
& \Lambda_{1}=\Omega^{+}\left[\begin{array}{c}
I_{n} \\
0
\end{array}\right], \Delta_{1}=-\left(I_{n+r_{1}+p}-\Omega \Omega^{+}\right)\left[\begin{array}{c}
I_{n} \\
0
\end{array}\right], \\
& \Lambda_{2}=\Omega^{+}\left[\begin{array}{c}
0 \\
I_{r_{1}+p}
\end{array}\right], \quad \Delta_{2}=-\left(I_{n+r_{1}+p}-\right. \\
& \left.\Omega \Omega^{+}\right)\left[\begin{array}{c}
0 \\
I_{r_{1}+p}
\end{array}\right],\left[\begin{array}{ll}
Q & F
\end{array}\right]=\left[\begin{array}{c}
R \\
C
\end{array}\right]^{-1}\left[\begin{array}{c}
K \\
I_{r_{1}+p}
\end{array}\right] \text { and }
\end{aligned}
$$
$K=\Lambda_{2}-Z_{1} \Delta_{2}$.

As in the above cases, the full order design can be obtained by following Algorithm IV-C.

\section{CONCLUSiON}

In this paper, we have presented the observer design for singular fractional-order systems. The obtained results unify the observers design of full, reduced and minimal orders. The conditions for the existence of these observers are given, necessary and sufficient conditions for their stability are derived in terms of linear matrix inequalities formulation with fractional-order $\alpha$ belonging to $0<\alpha<2$. 


\section{REFERENCES}

[1] L. Dai, Singular Control Systems, vol. 118 of Lecture Notes in Control and Information Sciences. New York: Springer-Verlag, 1989.

[2] M. Darouach, M. Zasadzinski, and M. Hayar, "Reduced-order observer design for descriptor systems with unknown inputs," IEEE Trans. Aut. Contr., vol. 41, pp. 1068-1072, 1996.

[3] M. Darouach and L. Boutat-Baddas, "Observers for a class of nonlinear singular systems," IEEE Trans. Aut. Contr., vol. 53, pp. 2627-2633, 2008.

[4] M. Darouach and M. Zasadzinski, "Data reconciliation in generalized linear dynamic systems," AICHE Journal, vol. 37, pp. 193-201, 1991.

[5] M. Boutayeb, M. Darouach, and H. Rafaralahy, "Generalized statespace observers for chaotic synchronization and secure communication," IEEE Trans. Circ. Syst. I : Fund. Theory \& Appli., vol. 49, pp. 345-349, 2002.

[6] M. Darouach, M. Zasadzinski, and S. Xu, "Full-order observers for linear systems with unknown inputs," IEEE Trans. Aut. Contr., vol. 39, pp. 606-609, 1994.

[7] M. Arcak and P. Kokotović, "Nonlinear observers : a circle criterion design and robustness analysis," Automatica, vol. 37, pp. 1923-1930, 2001.

[8] I. Podlubny, Fractional Differential Equations. New York: Academic, 1999.

[9] R. Hilfer, Applications of Fractional Calculus in Physics. Singapore: World Scientific Publishing, 2000.

[10] O. Heaviside, Electromagnetic Theory. New York: Chelsea Publishing Company, 3rd ed., 1971.

[11] N. Engheta, "On fractional calculus and fractional multipoles in electromagnetism," IEEE Trans. Antennas and Propagation, vol. 44, pp. 554-566, 1996.

[12] H. Sun, A. Abdelwahad, and B. Onaral, "Linear approximation of transfer function with a pole of fractional order," IEEE Trans. Aut. Contr., vol. 29, pp. 441-444, 1984.

[13] R. Bagley and R. Calico, "Fractional order state equations for the control of viscoelastically damped structures," J. Guidance, Contr. \& Dynamics, vol. 14, pp. 304-311, 1991.

[14] Y. Rossikhin and M. Shitikova, "Application of fractional derivatives to the analysis of damped vibrations of viscoelastic single mass system," Acta Mechanica, vol. 120, pp. 109-125, 1997.

[15] D. Luenberger, "Non-linear descriptor systems," J. Economic Dynamics, vol. 1, pp. 219-242, 1979.

[16] D. Matignon, "Stability results for fractional differential equations with applications to control processing," in Proc. IEEE-IMACS Syst. Man Cyber. Conf., (Lille, France), 1996.

[17] Y. Chen, H. Ahn, and I. Podlubny, "Robust stability check of fractional order linear time invariant systems with interval uncertainties," Signal Processing, vol. 86, pp. 2611-2618, 2006.

[18] I. Petráš, Y. Chen, and B. Vinagre, Unsolved problems in the mathematics of systems and control, vol. 38, ch. Robust stability test for interval fractional-order linear systems, pp. 208-210. Princeton: Princeton University Press, 2004. Ed. V. Blondel and A. Megretski.

[19] S. Das, Functional Fractional Calculus for System Identification and Controls. Heidelberg: Springer, 2008.

[20] I. Podlubny, "Geometric and physical interpretation of fractional integration and fractional differentiation," Fractional Calculus \& Applied Analysis, vol. 5, pp. 367-386, 2002.

[21] M. Chilali, Méthodes LMI pour l'Analyse et la Synthèse Multi-critère. $\mathrm{PhD}$ thesis, Université de Paris IX, Paris, France, 1996.

[22] O. Bachelier, Commande des Systèmes Linéaires Incertains : Placement de Pôles Robuste en $\mathcal{D}$-Stabilité. PhD thesis, Institut National des Sciences Appliquées de Toulouse, France, 1998.

[23] J. Sabatier, M. Moze, and C. Farges, "LMI conditions for fractional order systems," Computers \& Mathematics with Applications, vol. 59, pp. 1594-1609, 2010.

[24] D. Matignon, "Generalized fractional differential and difference equations : stability properties and modelling issues," in Proc. Mathematical Theory of Networks and Systems Symposium, (Padova, Italy), 1998.

[25] J. Sabatier, M. Moze, and C. Farges, "On stability of fractional order systems," in Proc. IFAC Workshop on Fractional Differentiation and its Application, (Ankara, Turkey), 2008.

[26] M. Chilali, P. Gahinet, and P. Apkarian, "Robust pole placement in LMI regions," IEEE Trans. Aut. Contr., vol. 44, pp. 2257-2270, 1999.

[27] J. Lu and Y. Chen, "Robust stability and stabilization of fractionalorder interval systems with the fractional-order $\alpha$ : The $0<\alpha<1$ case," IEEE Trans. Aut. Contr., vol. 55, pp. 152-158, 2010.
[28] C. Farges, M. Moze, and J. Sabatier, "Pseudo-state feedback stabilization of commensurate fractional order systems," Automatica, vol. 46, pp. 1730-1734, 2010.

[29] D. Matignon and B. Andréa-Novel, "Some results on controllability and observability of finite-dimensional fractional differential systems," in Proc. Mathematical Theory of Networks and Systems Symposium, (Lille, France), 1996.

[30] D. Matignon and B. Andréa-Novel, "Observer-based for fractional differential systems," in Proc. IEEE Conf. Decision \& Contr., (San Diego, USA), 1997.

[31] M. Darouach, "Complements to full order observer design for linear systems with unknown inputs," Applied Mathematics Letters, vol. 22, pp. 1107-1111, 2009. 